Pacific Journal of Mathematic 


\title{
AN APPLICATION OF A NEWTON-LIKE METHOD TO THE EULER-LAGRANGE EQUATION
}

\author{
RICHARD A. TAPIA
}

It is known that any function which minimizes a functional of the form $J(y)=\int_{a}^{b} f\left(x, y, y^{\prime}\right)$ and satisfies prescribed boundary values must be a solution of the corresponding EulerLagrange equation: $f_{3}\left(x, y, y^{\prime}\right)-\int_{a}^{x} f_{2}\left(x, y, y^{\prime}\right)=c$. Let us call any equation of the form: $g\left(x, y, y^{\prime}\right)-\int_{a}^{x} h\left(x, y, y^{\prime}\right)=c$ a generalized Euler-Lagrange equation.

In this paper we propose a Newton-like method and show that this proposed method is general enough to enable us to construct solutions of the generalized Euler-Lagrange equation.

Let $X$ and $Y$ be Banach spaces, $\Omega$ an open subset of $X$ and $P: \Omega \rightarrow Y$. By $[X, Y]$ we mean the Banach space of all bounded linear operators with the usual operator norm, by $P^{\prime}$ the first derivative of $P$ and by $P^{\prime \prime}$ the second derivative of $P$. The class of all functions defined on $\Omega$ which have continuous derivatives up to and including order $n$ at each point of $\Omega$ is denoted by $C^{n}(\Omega)$. Distinct elements of $C^{n}(\Omega)$ may have totally distinct ranges depending on the application. The distinction between Gateaux and Fréchet is unnecessary since the derivatives will be continuous.

2. The weak Newton sequence. Let $H$ and $Y$ be Banach spaces, $\Omega$ a nonempty open subset of $H$ and $P: \Omega \rightarrow Y$.

Definition. Given $x_{0} \in \Omega$ the sequence $\left\{x_{n}\right\}_{0}^{\infty}$,

$$
x_{n+1}=x_{n}-\left[P^{\prime}\left(x_{n}\right)\right]^{-1} P\left(x_{n}\right),
$$

is called the Newton sequence for $x_{0}$ (with respect to $P$ ).

Definition. Given $x_{0}, \bar{x} \in \Omega$ the sequence $\left\{x_{n}\right\}_{0}^{\infty}$,

$$
x_{n+1}=x_{n}-\left[P^{\prime}(\bar{x})\right]^{-1} P\left(x_{n}\right),
$$

is called the modified Newton sequence for $x_{0}$ at $\bar{x}$ (with respect to $P$ ). When $x_{0}=\bar{x}$ we say simply the modified Newton sequence for $x_{0}$.

REMARK. The Newton sequences exist if and only if $P^{\prime}(\bar{x})$ and $P^{\prime}\left(x_{n}\right)$ exist and are invertible and $x_{n} \in \Omega$ for all $n$. 
Let $X$ and $Y$ be Banach spaces, $H$ a closed linear subspace of $X, \Omega$ a nonempty open subset of $H$ and $P: \Omega \rightarrow Y$.

REMARK. It is easy to verify that $\Omega$ is open in $X$ if and only if $H=X$.

Let $D$ be the set of all $x \in \Omega$ for which there exists an operator $\Gamma(x)$ satisfying the following conditions:

(i ) $\Gamma(x) \in\left[B_{x}, X\right]$, where $B_{x}$ is a closed linear subspace of $Y$ containing $P(\Omega)$;

(ii) $\Gamma(x) P(\Omega) \subset H$;

(iii) $\Gamma(x) P^{\prime}(x)=I: H \rightarrow H$.

REMARK. The fact that $\Gamma(x)$ is defined on $P^{\prime}(x)(H)$ and $\Gamma(x) P^{\prime}(x)$ is defined from $H$ into $H$ is a consequence of conditions (i) and (ii). The following lemma shows this to be true.

LEMma 2.1. If there exists an operator $\Gamma_{0}$ satisfying the following conditions:

(a) $\Gamma_{0} \in\left[B_{0}, X\right]$, where $B_{0}$ is a closed linear subspace of $Y$ containing $P(\Omega)$;

(b) $\Gamma_{0} P(\Omega) \subset H$;

then for all $x \in \Omega$ we have the following:

(i ) $P^{\prime}(x)(H) \subset B_{0}$;

(ii) $\Gamma_{0} P^{\prime}(x)(H) \subset H$.

Proof. Assume $x \in \Omega$ and $h \in H$. It follows from (a) since $\Omega$ is open in $H$ that for small $t$

$$
\frac{P(x+t h)-P(x)}{t} \in B_{0} .
$$

Therefore $P^{\prime}(x)(h) \in B_{0}$ and $P^{\prime}(x)(H) \subset B_{0}$. Similarly (a), (b) and the fact that $H$ is closed imply

$$
\Gamma_{0} P^{\prime}(x)(h)=\lim _{t \rightarrow 0}\left[\frac{\Gamma_{0} P(x+t h)-\Gamma_{0} P(x)}{t}\right] \in H .
$$

Definition. If $x \in D$, then $\Gamma(x)$ is called a left inverse for $P^{\prime}(x)$.

Remark. If $H=X$ and $B_{x}=Y$, then this is the usual notion of a left inverse.

Let $\Gamma(x)$ denote a left inverse for $P^{\prime}(x)$. 
Definition. Given $x_{0} \in \Omega$ the sequence $\left\{x_{n}\right\}_{0}^{\infty}$,

$$
x_{n+1}=x_{n}-\Gamma\left(x_{n}\right) P\left(x_{n}\right),
$$

is called the weak Newton sequence for $x_{0}$ (with respect to $P$ ).

Definition. Given $x_{0}, \bar{x} \in \Omega$ the sequence $\left\{x_{n}\right\}_{0}^{\infty}$,

$$
x_{n+1}=x_{n}-\Gamma(\bar{x}) P\left(x_{n}\right),
$$

is called the weak modified Newton sequence for $x_{0}$ at $\bar{x}$ (with respect to $P$ ). When $x_{0}=\bar{x}$ we say simply the weak modified Newton sequence for $x_{0}$.

REMARK. The weak Newton sequences exist if and only if $\Gamma(\bar{x})$ and $\Gamma\left(x_{n}\right)$ exist and $x_{n} \in \Omega$ for all $n$.

Lemma 2.2. If $x \in \Omega$ and $\left[P^{\prime}(x)\right]^{-1} \in[Y, H]$, then for any $\Gamma(x)$ satisfying (2.1) we have:

(i) $B_{x}=Y$;

(ii) $\Gamma(x)(Y)=H$;

(iii) $\Gamma(x)=\left[P^{\prime}(x)\right]^{-1}$.

Proof. Since $P^{\prime}(x)$ is onto, $Y=P^{\prime}(x)(H)$. By Lemma 2.1 $P^{\prime}(x)(H) \subset B_{x}$, therefore $Y=B_{x}$. Also $\Gamma(x) y=\Gamma(x) P^{\prime}(x)\left[P^{\prime}(x)^{-1} y\right]=$ $P^{\prime}(x)^{-1} y$ for all $y \in Y$, therefore $\Gamma(x)=\left[P^{\prime}(x)\right]^{-1}$.

REMARK. In general there are many weak (modified) Newton sequences for a particular point; however, by Lemma 2.2 if the (modified) Newton sequence exists, then any weak (modified) Newton sequence coincides with it.

ExAMPle. Let $P: R^{1} \rightarrow R^{2}$ be given by $P(x)=(3 x-3 / 2,2 x-1)$ hence $P^{\prime}(x)=(3,2)$. If $\Gamma_{0}: R^{2} \rightarrow R^{1}$ is given by

$$
\Gamma_{0}\left(x_{1}, x_{2}\right)=\left(a, \frac{1-3 a}{2}\right) \cdot\left(x_{1}, x_{2}\right)
$$

for any real $a$, then $\Gamma_{0} P(x)=x-1 / 2, \Gamma_{0} P^{\prime}(x)=1$, the identity map in $R^{1}$, and the weak (modified) Newton sequence for $x_{0}$ is given by

$$
x_{n+1}=x_{n}-\Gamma_{0} P\left(x_{n}\right)=\frac{1}{2}
$$

for all $n$. Notice that $P(1 / 2)=(0,0)$.

REMARK. In this example there are an uncountable number of 
left inverses for $P^{\prime}(x)$; also the (modified) Newton sequence for $x_{0}$ does not exist.

3. Convergence theorems. As before let $X$ and $Y$ be Banach spaces, $H$ a closed linear subspace of $X, \Omega$ a nonempty open subset of $H$ and $P: \Omega \rightarrow Y$. If for each $x \in \Omega$ there exists an operator $\Gamma(x)$ satisfying conditions 2.1 and we let $B_{0}=\cap_{x \in \Omega} B_{x}$, where $B_{x}$ is the domain of $\Gamma(x)$, then $B_{0}$ is a closed linear subspace of $Y$ containing $P(\Omega)$; consequently by restricting $\Gamma(x)$ to $B_{0}$ we may consider $\Gamma: \Omega \rightarrow$ $\left[B_{0}, X\right]$.

\section{THEOREM 3.1. If}

(i) there exists $x^{*} \in \Omega$ such that $P\left(x^{*}\right)=0$,

(ii) for each $x \in \Omega$ there exists $\Gamma(x)$ satisfying conditions (2.1) and $\Gamma: \Omega \rightarrow\left[B_{0}, X\right] \in C^{\circ}(\Omega)$

then, if $P \in C^{1}(\Omega)$, given $0<\alpha<1$ there exists a neighborhood of $x^{*}$ contained in $\Omega$ such that for any 2 points $x_{0}$ and $\bar{x}$ in this neighborhood the weak modified Newton sequence for $x_{0}$ at $\bar{x}$ exists and converges to $x^{*}$; we also have

$$
\left\|x^{*}-x_{n}\right\| \leqq \frac{\alpha^{n}}{1-\alpha}\left\|x_{1}-x_{0}\right\| .
$$

Furthermore, if $P \in C^{2}(\Omega)$, then there exists a neighborhood of $x^{*}$ contained in $\Omega$ such that if $x_{0}$ is any point in this neighborhood a weak Newton sequence for $x_{0}$ exists and converges quadratically to $x^{*}$, i.e., there exists a constant $M$ such that

$$
\left\|x^{*}-x_{n+1}\right\| \leqq M\left\|x^{*}-x_{n}\right\|^{2} .
$$

Proof. For $x \in \Omega$ let $T(x)=I-\Gamma(x) P^{\prime}\left(x^{*}\right)$. Given $0<\alpha<1$ there exists $\delta>0$ such that $\left\{x \mid\left\|x-x^{*}\right\| \leqq \delta\right\} \subset \Omega$ and

$$
\|T(x)\|=\left\|T(x)-T\left(x^{*}\right)\right\|<\alpha
$$

whenever $\left\|x-x^{*}\right\| \leqq \delta$. Now if $\left\|\bar{x}-x^{*}\right\| \leqq \delta$ and $S=I-\Gamma(\bar{x}) P$, then

(i ) $S\left(x^{*}\right)=x^{*}$ and

(ii) $\left\|S^{\prime}\left(x^{*}\right)\right\|<\alpha$.

Let $\left\|S^{\prime}\left(x^{*}\right)\right\|=\alpha_{0}$, then there exists $0<\delta_{0} \leqq \delta$ such that

$$
\left\|S^{\prime}(x)-S^{\prime}\left(x^{*}\right)\right\| \leqq \alpha-\alpha_{0}
$$

whenever $x \in \Omega_{0}=\left\{x \mid\left\|x-x^{*}\right\| \leqq \delta_{0}\right\}$. Clearly $\Omega_{0}$ is closed, convex and contained in $\Omega$. Also

(i ) $\left\|S^{\prime}(x)\right\| \leqq \alpha$ whenever $x \in \Omega_{0}$ and 
(ii ) $\quad S\left(\Omega_{0}\right) \subset \Omega_{0}$;

since if $x \in \Omega_{0}$,

$$
\begin{aligned}
\left\|S(x)-x^{*}\right\| & =\left\|S(x)-S\left(x^{*}\right)\right\| \\
& \leqq\left\|S^{\prime}\left(x+\theta\left(x^{*}-x\right)\right)\right\|\left\|x-x^{*}\right\| \quad 0<\theta<1 \\
& \leqq \alpha\left\|x-x^{*}\right\| \leqq \delta_{0} .
\end{aligned}
$$

The first part of this theorem now follows from a well-known fixed point theorem $[4,661]$. The latter part of the theorem is a consequence of the following Banach space inequality [3, pp. 162-163].

$$
\left\|P(x+\Delta x)-P(x)-P^{\prime}(x)(\Delta x)\right\| \leqq \frac{1}{2}\left\|P^{\prime \prime}(x+\theta \Delta x)\right\|\|\Delta x\|^{2},
$$

$0<\theta<1$. There clearly exists $K$ and $\delta>0$ such that $\left\|P^{\prime \prime}(x)\right\| \leqq K$ and $\|\Gamma(x)\| \leqq K$, whenever $\left\|x^{*}-x\right\| \leqq \delta$. If $\left\|x^{*}-x\right\| \leqq \delta$, then from (3.1) with $\Delta x=x^{*}-x$ we obtain

$$
\left\|x^{*}-[x-\Gamma(x) P(x)]\right\| \leqq M\left\|x^{*}-x\right\|^{2}
$$

where $M=\frac{1}{2} K^{2}$. Choose $0<\delta_{0}<\min \left(M^{-1}, \delta\right)$ and such that

$$
\Omega_{0}=\left\{x \mid\left\|x^{*}-x\right\| \leqq \delta_{0}\right\} \subset \Omega .
$$

We now show if $X_{n} \in \Omega_{0}$, then

$$
\begin{aligned}
\left\|x^{*}-x_{n+1}\right\| & \leqq M\left\|x^{*}-x_{n}\right\|^{2} \\
& \leqq\left\|x^{*}-x_{0}\right\|\left(M\left\|x^{*}-x_{0}\right\|\right)^{2^{n+1}-1} \leqq \delta_{0} ;
\end{aligned}
$$

consequently $x_{n+1} \in \Omega_{0}$. For $n=0$ inequality (3.3) is just (3.2) with $x=x_{0}$. If we assume (3.3) holds for $n<k$, then a direct application of (3.2) shows (3.3) holds for $n=k$, and consequently for all $n$. This proves the theorem.

Consider $\Gamma_{0}: B_{0} \rightarrow X$, where $B_{0}$ is a closed linear subspace of $Y$ containing $P(\Omega)$, satisfying:

$$
\begin{array}{ll}
\text { (i) } & \Gamma_{0} \in\left[B_{0}, X\right] \text {; } \\
\text { (ii) } & \Gamma_{0} P(\Omega) \subset H \text {. }
\end{array}
$$

CoRollary 3.1. Given $x_{0} \in \Omega$, if there exists

(i) $x^{*} \in \Omega$ such that $P\left(x^{*}\right)=0$, and

(ii) $\Gamma_{0}$ satisfying (3.4) and such that $\Gamma_{0} P^{\prime}\left(x_{0}\right): H \rightarrow H$ is invertible,

then if $x_{0}$ is sufficiently near $x^{*}$ and $P \in C^{2}(\Omega)$ a weak (modified) Newton sequence for $x_{0}$ exists and converges to $x^{*}$. Furthermore $a$ left inverse for $P^{\prime}(x)$ is given by $\Gamma(x)=\left[\Gamma_{0} P^{\prime}(x)\right]^{-1} \Gamma_{0}$.

Proof. Use Theorem 3.1 on $Q=\Gamma_{0} P$. 
4. A general problem. Given an interval $[a, b]$ let

$$
\begin{aligned}
& X=\left\{y \in C^{0}[a, b] \mid y:[a, b] \rightarrow R^{1}\right\}, \\
& H=\left\{h \in X \mid \int_{a}^{b} h=0\right\}, \\
& Y=\left\{y \in C^{1}[a, b] \mid y:[a, b] \rightarrow R^{1}\right\}, \\
& K=\left\{y \in Y \mid y(a)=\mu_{1} \text { and } y(b)=\mu_{2}\right\}, \\
& B=[a, b] \times R^{2}, \\
& \|y\|_{X}=\sup \{\mid y(t) \| t \in[a, b]\} \text { for } y \in X, \text { and } \\
& \|y\|_{Y}=\sup \left\{|y(t)|,\left|y^{\prime}(t)\right| \mid t \in[a, b]\right\} \text { for } y \in Y .
\end{aligned}
$$

ReMARK. If $X, Y$ and $H$ are given by (4.1), then $X$ and $Y$ are Banach spaces and $H$ is a closed linear subspace of $X$.

Given continuous $\varphi: B \rightarrow R^{1}$ and $y_{0} \in K$ define

$$
\bar{Q}: Y \rightarrow X, \text { and } Q: X \rightarrow X
$$

as follows

$$
\begin{gathered}
\bar{Q}(y)(x)=\varphi\left(x, y(x), y^{\prime}(x)\right) \text { for } y \in Y ; \\
Q(y)=\bar{Q}\left(y_{0}+\int_{a}^{x} y\right) \text { for } y \in X .
\end{gathered}
$$

It is clear that if $y \in X$, then $y_{0}+\int_{a}^{x} y \in Y$. Assume $Q$ satisfies the following two conditions:

(i ) $Q \in C^{2}[X]$;

(ii) $\left[Q^{\prime}(0)\right]^{-1} \in[X, X]$.

Define $P: H \rightarrow X$ by

$$
P(h)=Q(h)-\frac{\int_{a}^{b} \Gamma_{0} Q(h)}{\int_{a}^{b} \Gamma_{0}(1)} \text { for } h \in H,
$$

where $\Gamma_{0}=\left[Q^{\prime}(0)\right]^{-1}$ and 1 is the constant function 1 .

Lemma 4.1. If $\bar{Q}$ is given by (4.2) and $P$ by (4.5), then the following two problems are equivalent:

Problem I. Find $y \in K$ such that $\bar{Q}(y)$ is constant;

Problem II. Find $h \in H$ such that $P(h)=0$.

Proof. If $y \in K$ and $\bar{Q}(y)$ is constant, then $h=y^{\prime}-y_{0}^{\prime} \in H$ and 
$Q(h)=\bar{Q}\left(y_{0}+\int_{a}^{x}\left(y^{\prime}-y_{0}^{\prime}\right)\right)$ is constant; therefore $P(h)=0$. Now if $h \in H$ and $P(h)=0$, then $Q(h)=\bar{Q}\left(y_{0}+\int_{a}^{x} h\right)$ is constant; therefore $y=$ $y_{0}+\int_{a}^{x} h \in K$ and $\bar{Q}(y)$ is constant.

Lemma 4.2. If $\Gamma_{0}$ and $P$ are given by (4.5), then $\Gamma_{0}$ is a left inverse for $P^{\prime}(0)$.

Proof. We show $\Gamma(0)=\Gamma_{0}$ satisfies (2.1) for $x=0$. If $B_{0}=X$ and $\Omega=H$, then (i) of (2.1) holds. If $h \in H$, then from (4.5) we obtain,

$$
\int_{a}^{b} \Gamma_{0} P(h)=0
$$

and (ii) of (2.1) holds. By differentiating (4.5) at the origin we see that

$$
\Gamma_{0} P^{\prime}(0)(h)=h \quad \text { for } \quad h \in H .
$$

Therefore $\Gamma_{0} P^{\prime}(0)=I: H \rightarrow H$ and the lemma is proved.

Let $y^{*} \in Y$ be a solution of Problem I and $h^{*} \in X$ the corresponding solution of Problem II.

Lemma 4.3. For given $y_{0}$ in (4.3)

$$
\left\|h^{*}\right\|_{X} \leqq\left\|y_{0}-y^{*}\right\|_{Y} \text {. }
$$

Proof. The proof follows from (4.1) and Lemma 4.1.

REMARK. If Problem I has a solution and the given $y_{0}$ in (4.3) is sufficiently near this solution, then by Lemmas 4.1, 4.2, 4.3 and Corollary 3.1 with $\Gamma_{0}=\left[Q^{\prime}(0)\right]^{-1}, x_{0}=0, \Omega=H, Y=X$ and $H, X$ and $P$ given by (4.1) and (4.5) both the weak Newton and weak modified Newton sequences for $x_{0}$ exist and can be used to obtain this solution. In addition the weak modified Newton sequence can still be used if we only have $Q \in C^{1}[H]$.

5. A variation of the weak Newton sequence. If $\Gamma_{0}$ and $P$ are given by (4.5) and $h_{n}$ is the $n^{\text {th }}$ term in the weak Newton sequence for $h_{0}=0$, then by Corollary 3.1 a left inverse for $P^{\prime}\left(h_{n}\right)$ is given by

$$
\Gamma\left(h_{n}\right)=\left[\Gamma_{0} P^{\prime}\left(h_{n}\right)\right]^{-1} \Gamma_{0} .
$$

This operator may be difficult to evaluate; we therefore consider the 
following variation of the weak Newton sequence for Problem II.

For $Q$ given by (4.3), assume the following conditions hold:

$$
\begin{aligned}
& \text { (i ) } Q \in C^{2}[X] ; \\
& \text { (ii) }\left[Q^{\prime}(x)\right]^{-1} \in[X, X] \text { for all } x \in X .
\end{aligned}
$$

Inherent in the above assumption is the requirement that a procedure for evaluating $\left[Q^{\prime}(x)\right]^{-1}$ is known. Let

$$
\Gamma(x)=\left[Q^{\prime}(x)\right]^{-1} \text { for } x \in X
$$

and define $h_{n} \in H$ and $P_{n}: H \rightarrow X$ recursively by

$$
\begin{aligned}
& h_{0}=0 \\
& P_{0}(h)=Q(h)-\frac{\int_{a}^{b} \Gamma\left(h_{0}\right) Q(h)}{\int_{a}^{b} \Gamma\left(h_{0}\right)(1)} \text { for } h \in H, \text { and } \\
& h_{n+1}=h_{n}-\Gamma\left(h_{n}\right) P_{n}\left(h_{n}\right), \\
& P_{n+1}(h)=Q(h)-\frac{\int_{a}^{b} \Gamma\left(h_{n+1}\right) Q(h)}{\int_{a}^{b} \Gamma\left(h_{n+1}\right)(1)}, \text { for } n=0,1, \cdots .
\end{aligned}
$$

Lemma 5.1. If $\Gamma\left(h_{n}\right)$ and $P_{n}\left(h_{n}\right)$ are given by (5.2) and (5.3), then $\Gamma\left(h_{n}\right)$ is a left inverse for $P_{n}^{\prime}\left(h_{n}\right)$.

Proof. The proof of this lemma is the same as the proof of Lemma 4.2.

TheOREM 5.1. If (5.1) holds and the given $y_{0}$ in (4.3) is sufficiently close to a solution of Problem I, then the sequence $\left\{h_{n}\right\}_{0}^{\infty}$ given by (5.3) will converge quadratically to the corresponding solution of Problem II.

Proof. The proof of this theorem is essentially the same as the latter part of the proof of Theorem 3.1.

Lemma 5.2. For $y_{0}$ used in (4.3) and $\left\{h_{n}\right\}_{0}^{\infty}$ given by (5.3), Problem I is equivalent to the following problem:

Problem III. Find $y_{0} \in K$ such that the sequence $\left\{h_{n}\right\}_{0}^{\infty}$ converges.

Proof. If $y$ is a solution to Problem I then by Theorem 5.1 we need only pick $y_{0} \in K$ near $y$. Now assume $\left\{h_{n}\right\}_{0}^{\infty}$ converges to $h$, then $\Gamma\left(h_{n}\right) P_{n}\left(h_{n}\right) \rightarrow 0$ and since $\Gamma^{-1}(h)=Q^{\prime}(h)$ exists we have $P_{n}\left(h_{n}\right) \rightarrow 0$ or $Q(h)$ is constant, Also, since $H$ is closed, $h \in H$; by Lemma $4.1 y=$ 
$y_{0}+\int_{a}^{x} h$ solves Problem 1

REMARK. We have shown that whenever the sequence $\left\{h_{n}\right\}_{0}^{\infty}$ given by (5.3) converges, it converges quadratically to a solution of Problem II and consequently gives a solution to Problem I.

6. The generalized Euler-Lagrange equation. Let $X, H, Y, K$ and $B$ be given by (4.1). In the calculus of variations one is interested in finding $y \in K$ such that for all $x \in[a, b]$

$$
f_{3}\left(x, y(x), y^{\prime}(x)\right)-\int_{a}^{x} f_{2}\left(x, y(x), y^{\prime}(x)\right)=c,
$$

where $f: B \rightarrow R^{1}, f_{i}$ denotes the $i^{\text {th }}$ partial derivative of $f$ and $c$ is an unknown constant. Historically equation (6.1) has been called the Euler-Lagrange equation.

For $g, h: B \rightarrow R^{1}$, we would like to find $y \in K$ such that for all $x \in[a, b]$

$$
g\left(x, y(x), y^{\prime}(x)\right)-\int_{a}^{x} h\left(x, y(x), y^{\prime}(x)\right)=c .
$$

We are therefore interested in solving Problem I (4.6) with $\bar{Q}: Y \rightarrow X$ given by:

$$
\bar{Q}(y)(x)=g\left(x, y(x), y^{\prime}(x)\right)-\int_{a}^{x} h\left(x, y(x), y^{\prime}(x)\right) .
$$

Since equation (6.1) is a special case of equation (6.2), we call (6.2) the generalized Euler-Lagrange equation.

Given $f_{i, j} \in X, i=1,2, \cdots, n$ and $j=1,2, \cdots, 5$, define the integral operator $L: X \rightarrow X$ as follows: for $u \in X$ let

$$
\begin{aligned}
\mathrm{L}(u)(x)= & \sum_{i=1}^{n}\left[f_{i, 1}(x) \int_{a}^{x} f_{i, 2}(y) u(y) d y\right. \\
& \left.+f_{i, 3}(x) \int_{a}^{x}\left(f_{i, 4}(y) \int_{a}^{y} f_{i, 5}(t) u(t) d t\right) d y\right] .
\end{aligned}
$$

Theorem 6.1. If $L$ is given by (6.4) and $\lambda$ is any constant, then (i) $L \in[X, X]$,

(ii) for all $u \in X$ the series

$$
\|u\|+\|\lambda L(u)\|+\cdots+\left\|\lambda^{n} L^{n}(u)\right\|+\cdots
$$

is convergent.

Furthermore,

(iii) for all $\varphi \in X$ the equation 


$$
f=\varphi+\lambda L(f),
$$

has a unique solution in $X$ which can be obtained by iterating (6.5) beginning with an arbitrary element of $X$; finally

(iv) the operator $T=I-\lambda L$ has a continuous inverse which can be evaluated by iteration.

Proof. The proof of (i) is immediate. Since (iii) proceeds directly from (ii) and (iv) follows from (i) and (iii) we will only prove (ii).

For $u \in X$ let $K(u)(x)=\int_{a}^{x} u$. Then there exists a constant $B$ such that for $x \in[a, b]$

$$
|L(u)(x)| \leqq B\left(K+K^{2}\right)(|u|)(x) \leqq B\|u\|\left(K+K^{2}\right)(1)(x) .
$$

Assume for $1 \leqq n \leqq k$

$$
\left|L^{n}(u)(x)\right| \leqq B^{n}\left(K+K^{2}\right)^{n}(|u|)(x) \leqq B^{n}\|u\|\left(K+K^{2}\right)^{n}(1)(x),
$$

then

$$
\begin{aligned}
& \left|L^{k+1}(u)(x)\right|=\left|L^{k}(L(u))(x)\right| \leqq B^{k}\left(K+K^{2}\right)^{k}(|L(u)|)(x) \\
& \leqq B^{k+1}\left(K+K^{2}\right)^{k+1}(|u|)(x) \leqq B^{k+1}\|u\|\left(K+K^{2}\right)^{k+1}(1)(x)
\end{aligned}
$$

and by induction (6.7) holds for all $n$.

If we let $M=\max (1,\|u\|,|\lambda| B)$ and denote $K^{n}(1)(x)$ by $K^{n}$, then

$$
\begin{aligned}
\sum_{n=0}^{\infty}\left|\lambda^{n} L^{n}(u)(x)\right| & \leqq \sum_{n=0}^{\infty} M^{n+1}\left(K+K^{2}\right)^{n} \leqq \sum_{n=0}^{\infty} \sum_{j=0}^{n} M^{n+j+1}\left(\begin{array}{c}
n \\
j
\end{array}\right) K^{n+j} \\
& =\sum_{m=0}^{\infty} A_{m} M^{m+1} \frac{(x-a)^{m}}{m !}
\end{aligned}
$$

where $A_{m}=\sum_{i=0}^{[m / 2]}\left(\begin{array}{c}m-i \\ i\end{array}\right)$ and $[x]$ denotes the largest integer less than or equal to $x$. The first inequality in (6.8) follows from (6.7) and the second inequality is a direct application of the binomial theorem. The equality in (6.8) is justified by observing that the coefficients of $K^{m}$ for arbitrary but fixed $m$ are of the form $M^{n+j+1}\left(\begin{array}{c}n \\ j\end{array}\right)$ where $j \leqq$ $n$ and $n+j=m$ and also that $K^{m}=(x-a)^{m} / m$ !. It is not difficult to show that $A_{m}$ satisfies the difference equation $A_{m+1}=A_{m}+A_{m-1}$; consequently the radius of convergence of the last series in (6.8) is infinite. This proves (ii) and the theorem follows.

Lemma 6.1. For $g, h \in C^{\circ}(B)$ let $Q$ be given by (4.3) and (6.3). If $g, h \in C^{n}(B)$ then $Q \in C^{n}(X)$, for $n=0,1,2$. 
Proof. The proof is straightforward although somewhat lengthy [6].

If $X, K$ and $B$ are given by (4.1) and $y_{0} \in K$, then for continuous $g: B \rightarrow R^{1}$ define $\bar{g}: X \rightarrow X$ as follows:

$$
\bar{g}(y)(x)=g\left(x, y_{0}(x)+\int_{a}^{x} y(t), y_{0}^{\prime}(x)+y(x)\right)
$$

for $y \in X$ and $x \in[a, b]$.

THEOREM 6.2. For $g, h: B \rightarrow R^{1}$, if

(a) $g, h \in C^{2}(B)$, and

(b) $\bar{g}_{3}(y) \neq 0$ for $y \in X$

and if $Q: X \rightarrow X$ is given by (4.3) and (6.3) then

(i ) $Q \in C^{2}[X]$,

(ii) $\left[Q^{\prime}(y)\right]^{-1} \in[X, X]$ for all $y \in X$ and can be evaluated by iteration.

Proof. Part (i) follows from Lemma 6.1. A direct calculation shows that

$$
Q^{\prime}(y)(\eta)=\bar{g}_{3}(y) \eta+\bar{g}_{2}(y) \int_{a}^{x} \eta-\int_{a}^{x}\left(\bar{h}_{2}(y) \int_{a}^{x} \eta\right)-\int_{a}^{x} \bar{h}_{3}(y) \eta,
$$

for $y, \eta \in X$. The subscripts on $\bar{g}$ and $\bar{h}$ denote partial derivatives. If we let

$$
\begin{gathered}
f=\frac{1}{\bar{g}_{3}(y)} \\
f_{11}=f \cdot \bar{g}_{2}(y), f_{12}=1, f_{13}=-f, f_{14}=\bar{h}_{2}(y), f_{15}=1, \\
f_{21}=-f, f_{22}=\bar{h}_{3}(y) \text { and } f_{23}=f_{24}=f_{25}=0, \text { then } \\
T(\eta)=\eta+L(\eta)=f \cdot Q^{\prime}(y)(\eta)
\end{gathered}
$$

where $y, \eta \in X$ and $L: X \rightarrow X$ is given by (6.4) and (6.9) with $n=2$ and $T: X \rightarrow X$ is given by (6.6) with $\lambda=-1$. Given $\varphi, y \in X$ constructing $\eta=\left[Q^{\prime}(y)\right]^{-1}(\varphi)$ is equivalent to solving

$$
T(\eta)=f \varphi \quad \text { for } \quad \eta \in X,
$$

hence $\eta=T^{-1}(f \varphi)$ and according to Theorem 6.1, $\eta$ is given by

$$
\eta=f \varphi-L(f \varphi)+L^{2}(f \varphi)-L^{3}(f \varphi)+\cdots .
$$

REMARKs. It follows that the theory developed in $\S 1$ through $\S 5$ can be used to solve the generalized Euler-Lagrange equation. In 
the calculus of variations condition (b) above is called the strengthened Legendre condition.

Theorem 6.2 includes the problem of finding $y \in K$ such that

$$
y^{\prime \prime}=h\left(x, y, y^{\prime}\right) \quad \text { where } \quad h \in C^{2}(B) .
$$

If $g: B \rightarrow R^{1}$ is defined by

$$
g(x, y, z)=z, \text { then } \bar{g}_{3}(y)=1 \neq 0
$$

and $g \in C^{2}(B)$, therefore Theorem 6.2 holds. Now if

$$
y^{\prime}-\int_{a}^{x} h\left(x, y, y^{\prime}\right)=\text { constant },
$$

then since two of the three terms in (6.11) are differentiable, the third must also be, giving (6.10).

The author would like to thank the referee for many helpful comments and suggestions.

\section{REFERENCES}

1. J. E. Dennis, Variations on Newton's method, Ph. D. Dissertation, University of Utah, 1966.

2. I. M. Gelfand and S. V. Fomin, Calculus of variations, Prentice-Hall, Inc., New Jersey, 1963.

3. W. L. Hart and T. S. Motzkin, A composite Newton-Raphson gradient method for the solution of systems of equations, Pacific J. Math. 6 (1956), 691-707.

4. L. V. Kantorovich, Functional analysis and applied mathematics, translated from the Russian by C. D. Benster, National Bureau of Standards Report 1509, 1948.

5. L. V. Kantorovich and G. P. Akilov, Functional analysis in normed spaces, Pergamon Press, New York, 1964.

6. M. L. Stein, On methods of obtaining solutions of fixed end point problems in the calculus of variations, Ph. D. Dissertation, University of California, Los Angeles, 1950.

7. R. A. Tapia, A generalization of Newton's method with an application to the Euler-Lagrange equation, Ph. D. Dissertation, University of California, Los Angeles, 1967.

8. A. E. Taylor, Introduction to functional analysis, John Wiley and Sons, Inc., New York, 1963.

Received February 12, 1968. The research on this paper was sponsored by the Office of Naval Research under Contract NONR 233(76). Reproduction in whole or in part is permitted for any purpose of the United States Government; and represents a part of the author's doctoral dissertation directed by Professor C. B. Tompkins.

UNiversity of CALIFornia, LOS ANGEles

AND

Mathematics Research Center, Univesity of Wisconsin 



\section{Pacific Journal of Mathematics \\ Vol. 29, No. 1 \\ May, 1969}

Jorge Alvarez de Araya, A Radon-Nikodým theorem for vector and operator

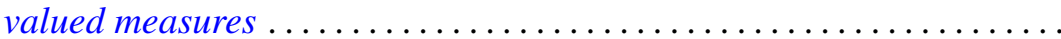

Deane Eugene Arganbright, The power-commutator structure of finite

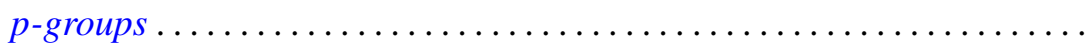

Richard Eugene Barlow, Albert W. Marshall and Frank Proschan, Some

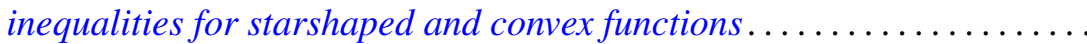

David Clarence Barnes, Some isoperimetric inequalities for the eigenvalues

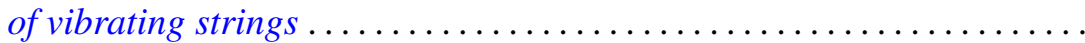

David Hilding Carlson, Critical points on rim-compact spaces ...........

Allan Matlock Weber Carstens, The lattice of pretopologies on an arbitrary

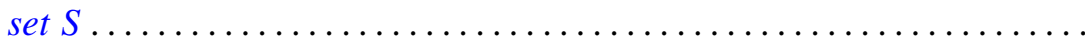

S. K. Chatterjea, A bilateral generating function for the ultraspherical

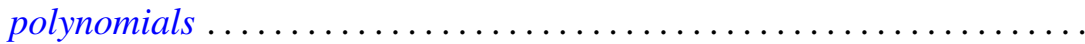

Ronald J. Ensey, Primary Abelian groups modulo finite groups ......... 77

Harley M. Flanders, Relations on minimal hypersurfaces ............ 83

Allen Roy Freedman, On asymptotic density in n-dimensions........... 95

Kent Ralph Fuller, On indecomposable injectives over artinian rings...... 115

George Isaac Glauberman, Normalizers of p-subgroups in finite groups . . . 137

William James Heinzer, On Krull overrings of an affine ring ........... 145

John McCormick Irwin and Takashi Ito, A quasi-decomposable abelian group without proper isomorphic quotient groups and proper isomorphic subgroups.

Allan Morton Krall, Boundary value problems with interior point boundary conditions

John S. Lowndes, Triple series equations involving Laguerre

polynomials

Philip Olin, Indefinability in the arithmetic isolic integers

Ki-Choul Oum, Bounds for the number of deficient values of entire functions whose zeros have angular densities..

R. D. Schafer, Standard algebras ....................

Wolfgang M. Schmidt, Irregularities of distribution. III.

Richard Alfred Tapia, An application of a Newton-like method to the Euler-Lagrange equation 